\title{
Research on Rolling Force of Hot Rolling Strip Based on Improved Genetic Algorithm
}

\author{
Xingdong Li * \\ College of Mechanical Engineering, Yanshan University, Qinhuangdao, Hebei 066004, China \\ Ixd1975@ysu.edu.cn
}

Keywords: rolling force; hot strip; slipping friction; stagnant friction; genetic algorithm

Abstract. Rolling force model was developed in which the contact arc surface was adopted as slipping and stagnant friction. The positions of neutral plane, forward and backward slipping zone, and stagnant zone were computed with improved genetic algorithm. Rolling forces of hot strip were simulated, which agreed with measured ones from a hot strip plant.

\section{Introduction}

Friction in contact arc surface is an important factor influencing rolling force. Huang Changqing ${ }^{[1]}$ et al. developed a rolling force model in which radius of elastic flattened roll and stress state were considered and the assumption was that contact arc surface was full sticking friction ${ }^{[1-3]}$. Li Zhijie $^{[4]}$ et al. developed a rolling force model in which the influence of deformation rate on deformation resistance was considered and the assumption was that contact arc surface was full slipping friction.

However, slipping and stagnant frictions ${ }^{[5]}$ are contained when deformation zone length to average strip thickness ratio is 2 to 4 according to a hot rolling mill. No studies have investigated rolling force by assuming that the contact arc surface was slipping and stagnant friction.

In this paper, the contact arc surface was adopted as slipping and stagnant friction. The model for computing positions of neutral plane, slipping and stagnant zone was developed by genetic algorithm. A series of rolling force simulations were conducted to assess the validity of proposed models.

\section{Computation Model of Rolling Force}

Friction in contact arc surface were divided into backward slipping, stagnant and forward slipping zone, $n$ is neutral plane, as shown in Fig. 1. According to friction condition, two kinds of rolling forces per unit area $p_{x}$ and friction forces per unit area $t_{x}$ equations were described in the following.

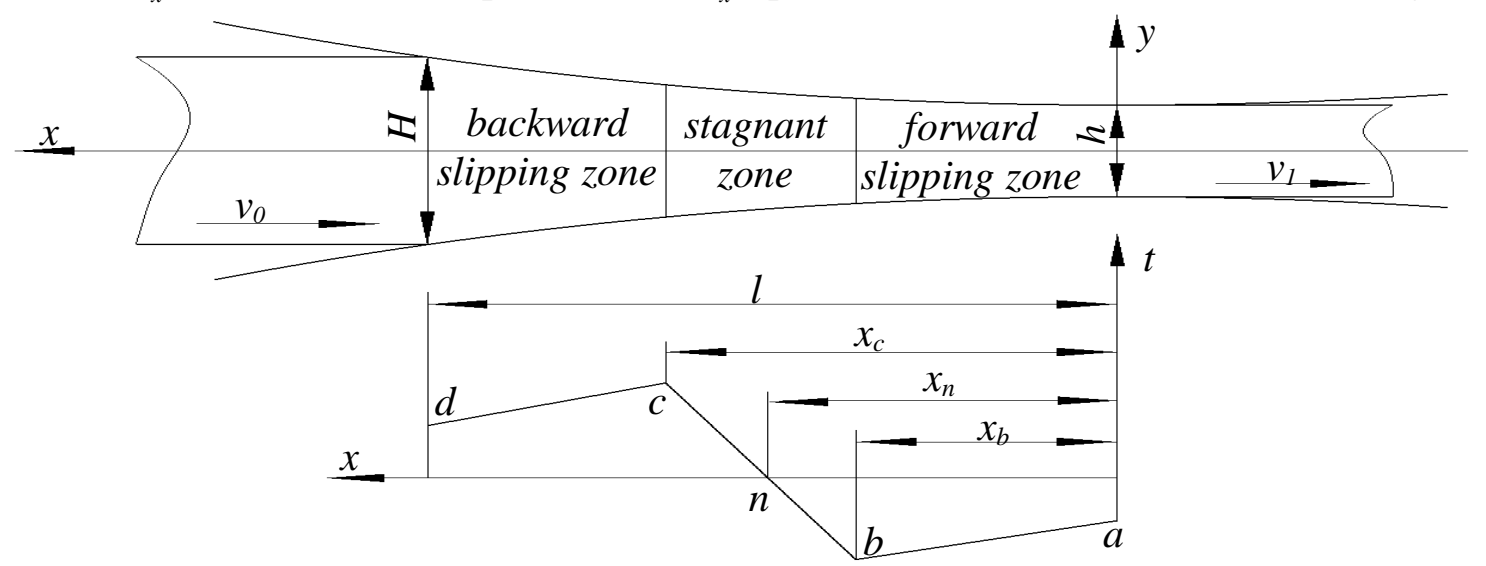

Fig. 1 Friction condition in contact arc surface

In forward slipping zone $p_{x}$ and $t_{x}$ can be determined as

$$
p_{x}=2 k\left(1-\frac{\sigma_{1}}{2 k}+\frac{\operatorname{tg} \theta_{a b}}{\mu}\right)\left(\frac{h_{x}}{h}\right)^{\mu / \operatorname{tg} \theta_{a b}}-\frac{2 k}{\mu} \operatorname{tg} \theta_{a b} .
$$


Where $k, \sigma_{1}$ and $\mu$ denote shear yield limit, exit tension, and friction coefficient, respectively; $h_{x}$ denote strip thickness at point $x$.

$$
t_{x}=\mu p_{x}
$$

In stagnant zone, $p_{x}$ and $t_{x}$ can be determined as

$$
p_{x}=p_{c}+\frac{t_{c}}{\left(h_{c}-h_{n}\right) \operatorname{tg} \theta_{b c}}\left(h_{c}-h_{x}-h_{n} \ln \frac{h_{c}}{h_{x}}\right)-2 k \ln \frac{h_{c}}{h_{x}} .
$$

Where $p_{c}$ and $h_{c}$ denote $p_{x}$ and $h_{x}$ at point $c$, respectively; $t_{c}$ denotes $t_{x}$ at point $c$ computed by Eq. 2, and $h_{n}$ denotes $h_{x}$ at point $n$.

$$
t_{x}=t_{c} \frac{h_{x}-h_{n}}{h_{c}-h_{n}}
$$

Strip width is $B$, then rolling force $P$ of hot strip can be determined as

$$
P=B \int_{0}^{l} p_{x} d x
$$

To computed $P$ with the above equations, $x_{b}, x_{n}$ and $x_{c}$ must be determined beforehand.

\section{Computation Model of $x_{b}, x_{n}$ and $x_{c}$}

At point $b, p_{x}$ can be computed by Eq. 1 and Eq. 3, and $t_{x}$ can be computed by Eq. 2 and Eq. 4 . Then, the following nonlinear equations can be obtained

$$
\left\{\begin{array}{l}
p_{b}^{1}-p_{b}^{3}=0 \\
t_{b}^{2}-t_{b}^{4}=0
\end{array}\right. \text {. }
$$

Where $p_{b}^{1}$ and $p_{b}^{3}$ denote $p_{x}$ at point $b$ computed by Eq. 1 and Eq. 3, respectively; $t_{b}^{2}$ and $t_{b}^{4}$ denote $t_{x}$ at point $b$ computed by Eq. 2 and Eq.4, respectively.

For solving nonlinear equations, traditional numerical methods are limited to functional continuity and derivability. However, genetic algorithm ${ }^{[6]}$ does not require differentiable function, and has the advantages of global and local search. Therefore, genetic algorithm was adopted in this paper.

Determination and Code of Computed Parameters. $x_{n}$ is an important parameter in strip rolling, which was adopted as an parameter. Stagnant zone length was equal to average strip thickness $h_{a}$, and $x_{b}$ was determined as another parameter. Then, $x_{c}$ could be determined. Real code can improve computational complexity of genetic algorithm by adopting true value of computed parameters. Therefore, $x_{n}$ and $x_{b}$ were coded by real.

Determination of Fitness Function. The solution of Eq. 6 by genetic algorithm made the differences between Eq. 1 and Eq. 3 and between Eq. 2 and Eq. 4 at point $b$ to be least. Rolling force is greatly more than friction force in hot strip rolling. Higher fitness individuals are easier to be inherited 
to next generation individuals. To ensure positive value of optimal object, fitness function $f(x)$ of genetic algorithm was determined as

$$
f(x)=\frac{1}{\left|\left(p_{b}^{1}-p_{b}^{3}\right) / p_{b}^{3}\right|+\left|\left(t_{b}^{2}-t_{b}^{4}\right) / t_{b}^{4}\right|} .
$$

Improved Fitness Function. Super value individuals can make rapidly local optimum solution in early genetic computation, and little difference individuals can make random research in lately genetic computation. To solve these problems, individuals were sequenced from large to small according to fitness value computed by Eq. 7, and new fitness function $F_{k}$ was determined as

$$
F_{k}=0.93^{(\lambda g)(k-1)}
$$

Where $k$ denotes sequence number of individual, $g$ denotes evolutional generation, and $\lambda$ denotes correction factor, which determines influence degree of evolutional generation on fitness value. $\lambda$ was adopted as 0.025 in this paper.

Genetic Computation Model. Selection operator is to select some better individuals to be reproduced to next generation. Crossover operator is to reproduce better individuals to next generation and construct new gene. Mutation operator is to change some genes of individual and then diversity of individuals is made. In this paper, proportional selection operator was adopted to construct parents, parents were made non uniform arithmetic crossover, and then Non uniform mutation operator was made.

\section{Validation}

A series of data related to hot strip rolling process was collected from the 7-stand hot rolling mill group. Taking one rolling strip as example, strip width was $1350 \mathrm{~mm}$ and strip thickness at entrance of pass one was $42 \mathrm{~mm}$. From pass one to seven, strip thicknesses at exit were 20.91, 12.76, 8.54, 6.06, 4.52, 3.75, and 3.37mm, and work roll diameters were 824.2, 747.1, 790.4, 630.2, 582.7, 627.6, $641.3 \mathrm{~mm}$.

Being close to exit, $x_{n}$ was adopted as $0.3 l$ to $0.49 l$. Stagnant zone is possibly asymmetry to neutral plane, therefore $x_{b}$ was adopted as $x_{n}-0.31 h_{a}$ to $x_{n}-0.65 h_{a}$. Individual number was adopted as 20 , crossover rate was adopted as 0.43 , and mutation rate was adopted as 0.029 .

The step to solve $x_{n}$ and $x_{b}$ by genetic algorithm was as following. The limit of individual value was computed. Twenty individuals were created within their limit values. After individual fitness was computed by Eq. 7, the individuals were sequenced from large to small, and then new individual fitness was computed by Eq. 8. Parents were generated by proportional selection, and made non uniform arithmetic crossover. Finally, by non uniform mutation, next generation was created to make cyclic computation. Until the evolution generation was two hundreds, genetic operator was finished. The best individual was adopted as solutions of Eq. 6. Values of $x_{b}, x_{n}, x_{c},\left(p_{b}^{1}-p_{b}^{3}\right) / p_{b}^{3}$ and $\left(t_{b}^{2}-t_{b}^{4}\right) / t_{b}^{4}$ are given in Table 1. 
Table.1 $\quad x_{b}, x_{n}, x_{c},\left(p_{b}^{1}-p_{b}^{3}\right) / p_{b}^{3}$ and $\left(t_{b}^{2}-t_{b}^{4}\right) / t_{b}^{4}$

\begin{tabular}{cccccccc}
\hline pass & 1 & 2 & 3 & 4 & 5 & 6 & 7 \\
\hline$x_{b}[\mathrm{~mm}]$ & 16.88 & 11.76 & 10.84 & 5.64 & 5.74 & 4.26 & 3.03 \\
\hline$x_{n}[\mathrm{~mm}]$ & 35.26 & 21.66 & 17.04 & 9.89 & 8.81 & 6.59 & 5.11 \\
\hline$x_{c}[\mathrm{~mm}]$ & 48.33 & 28.6 & 21.49 & 12.94 & 11.03 & 8.39 & 6.59 \\
\hline$\left(p_{b}^{1}-p_{b}^{3}\right) / p_{b}^{3}$ & $0.092 \% 0$ & $0.045 \% 0$ & $\begin{array}{c}-0.067 \\
\% 0\end{array}$ & $0.015 \% 0$ & $\begin{array}{c}-0.038 \\
\% o\end{array}$ & $\begin{array}{c}-0.029 \\
\% o\end{array}$ & $\begin{array}{c}-0.001 \\
\% o\end{array}$ \\
\hline$\left(t_{b}^{2}-t_{b}^{4}\right) / t_{b}^{4}$ & $0.112 \% 0$ & $\begin{array}{c}-0.103 \\
\% o\end{array}$ & $\begin{array}{c}-0.056 \\
\% o\end{array}$ & $0.085 \% 0$ & $\begin{array}{c}-0.064 \\
\% o\end{array}$ & $\begin{array}{c}-0.119 \\
\% o\end{array}$ & $\begin{array}{c}-0.033 \\
\% o\end{array}$ \\
\hline
\end{tabular}

From Table 1 it can be seen that stagnant zone was asymmetry to neutral plane, the distance between $x_{n}$ and $x_{b}$ was some more than that between $x_{n}$ and $x_{c}$. Values $\left(p_{b}^{1}-p_{b}^{3}\right) / p_{b}^{3}$ and $\left(t_{b}^{2}-t_{b}^{4}\right) / t_{b}^{4}$ were so small that they can be neglected, which showed reliability of the improved genetic algorithm for solving non nonlinear equations Eq. 6. On the basis of the above models, rolling forces of hot strip were simulated with $x_{b}, x_{n}$ and $x_{c}$ in Table 1 . Computed and measured rolling forces are shown in Fig. 2.

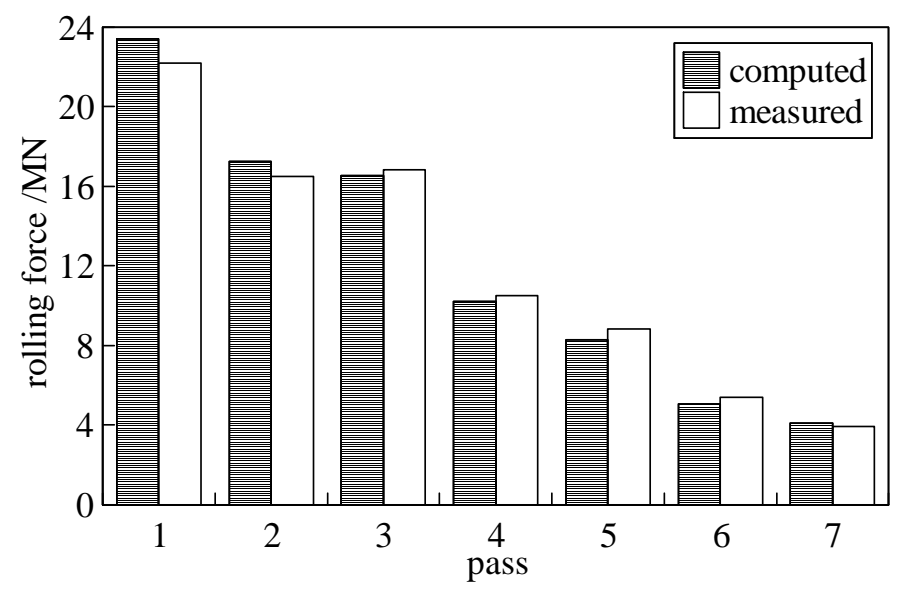

Fig. 2 Computed and measured rolling force

From Fig. 2 it can be seen that rolling force reduced with the increase of rolling pass because press value of strip reduced accordingly. The maximum errors between computed and measured rolling pressure were around $6 \%$ at pass 1, 5 and 6 , and the minimum error between computed and measured rolling pressure were around $2 \%$ at pass 3 . Computed rolling forces were in good agreement with measured ones, which showed reliability of the employed models.

\section{Conclusions}

According to deformation zone length to average strip thickness ratio in a hot strip plant, computed model of rolling force was developed where the contact arc surface was adopted as slipping and stagnant friction. Improved genetic algorithm model was developed to compute parameters in contact arc, with fitness function dynamically calibrated based on sequence and evolution generations. A series of process simulations were conducted, and comparison between the measured and predicted rolling force showed the reliability of the employed model that can be used to direct the prediction of rolling force during hot strip rolling.

\section{References}

[1]Huang Changqing, Deng Hua, Chen Jia, Hu Xinghua and Yang Shuangcheng: submitted to Journal of Procedia Engineering (2011) 
[2]Li Weigang,Tan Shubin,Li Jiabo and Li Long: submitted to Journal of Northeastern University (2011)

[3]Zhu Yu,Wang Tao. submitted to Machine Design and Manufacturing Engineering(2013)

[4]Li Zhijie, Peng Yan, Liu Hongmin, Wang Sufen and Xiao Lizi: submitted to Journal of China Mechanical Engineering (2013)

[5]Lian Jiachuang: submitted to Journal of Heavy Machinery (1975)

[6] Wang Xianbin, Shi Shuming, Liu Li and Jin Lisheng: submitted to Journal of China Mechanical Engineering (2014) 